\title{
An Array of Spherically Dimpled Scintillating Cells for a Calorimeter Integrated Readout Layer
}

\author{
G. Blazey, A. Dyshkant ${ }^{1}$, K. Francis, D. Hedin, E. Johnson, V. Zutshi \\ Northern Illinois University \\ DeKalb, Illinois 60115, USA \\ E-mail: dyshkantenicadd.niu.edu
}

\section{Lindenmeyer, A. Pla-Dalmau, E. Ramberg, J. Schellpfeffer}

Fermi National Accelerator Laboratory

Box 500, Batavia, Illinois 60510, USA

The CALICE collaboration is developing calorimetry for particle flow algorithm (PFA) based detectors. To measure particle shower development, a finely segmented calorimeter optimized for PFA must consist of millions of channels. A scintillator-based calorimeter with cells of size $30 \times 30 \times 3 \mathrm{~mm}^{3}$ readout with silicon photo-multipliers (SiPMs) in situ shows great promise. The construction and assembly challenges of a highly segmented scintillator-SiPM calorimeter could be greatly reduced with full integration of the readout electronics into active calorimeter layers. An integrated readout layer (IRL) comprised of a multi-channel electronics board instrumented with SiPMs paired to a solid uniform array of scintillator cells would eliminate much of the labor intensive production associated with the fiber readout cells. We discuss the development and performance of injection molded polystyrene arrays of $2 \times 2$ dimpled scintillator cells with a thickness of $3 \mathrm{~mm}$ and cell area of about $900 \mathrm{~mm}^{2}$. The spherically dimpled cells ensure uniformity of response for a SiPM positioned at the center of the cell. The performance of an array of molded cells is compared to an array of cells uniformly machined from a solid sheet of polyvinyltolulene cast scintillator with $3 \mathrm{~mm}$ thickness. To improve the performance of the molded array the cell dimple depth was optimized. The light output, uniformity, and cross talk of the molded and machined cells irradiated with a $\mathrm{Sr}-90$ source and measured with a SiPM in current mode are reported. Plans for initial particle beam tests of the complete IRL are also discussed.

Technology and Instrumentation in Particle Physics 2014

2-6 June, 2014

Amsterdam, the Netherlands

${ }^{1}$ Speaker 


\section{Introduction}

To obtain uniform response across scintillator tiles, the CALICE collaboration, for example, used a mirrored WLS fiber embedded into a grooved tile and readout with SiPM in situ [1,2]. We have previously reported [3] that spherically dimpled scintillating cells (with area about $900 \mathrm{~mm}^{2}$ and $5 \mathrm{~mm}$ thickness made of polyvinyltolulene cast scintillator) readout directly with a silicon photo-detector with active area about $1 \mathrm{~mm}^{2}$ has a highly uniform response when irradiated with a collimated Sr-90 source. The photo detector was located at the center of the dimple on the dimple side of the cell. The entrance window of the photo detector was flush with the flat surface of the dimpled side. This approach has also been proven for different $120 \mathrm{GeV}$ proton beam incidences for a set of single cells with a mechanically produced spherical dimple of about $70 \%$ depth in cast and extruded scintillator materials $[4,5]$.

In calorimetry for PFA based detectors, the high production and assembly costs for a large number of single scintillating tiles (each with WLS fiber, mirror, photo-detector, and possible wrapping) can be avoided by instrumenting a full electronics board (including SiPMs) and connecting that board to an active layer (a solid uniform array consisting of many small scintillator tiles fabricated and joined together). Such "direct coupling" eliminates wavelength shifting fibers but without careful modification, the response over a flat cell will be nonuniform. Experiments indicate that a spherical dimple in the scintillator tile improves cell response uniformity. The possible simplifications of an IRL are attractive, but the concept requires development and feasibility tests.

\section{Molded and Mechanically Produced Arrays of Cells with Spherical Dimples.}

For the IRL, the electronics board can be produced by industry. Production of a large array of $3 \mathrm{~mm}$ thick scintillating cells (CALICE AHCAL [1]) with spherical dimples needs additional exploration. We developed and tested molded polystyrene arrays of 2 x 2 dimpled cells, each cell with about $9 \mathrm{~cm}^{2}$ area. These are compared to mechanically milled cell arrays. Such arrays can have dimensions up to $1.5 \mathrm{~m}$ wide, $3.6 \mathrm{~m}$ long, and $10 \mathrm{~mm}$ thick. For the first tests an array of $9 \times 11$ cells was produced. Arrays can also be of size $12 \times 12,12 \times 24$, or $12 \times$ 36 cells, if those designs are practical. The response of polystyrene cells produced by injection molding was not as "flat" or uniform as cells with mechanically produced dimples.

Accordingly, the depth of the dimple in the injection molded cells was increased to get a flatter response. For the molded single cell the flattest uniformity was at a dimple depth of about $90 \%$ of the cell thickness. The $90 \%$ depth is near the physical limit of the cell, but there was still no flat response and no indications of overcompensation that we observed with mechanically produced dimples when the depth of dimple was more than $70 \%$ of the cell thickness [3]. A depth of $90 \%$ was used in a molded array of four square $(2 \times 2)$ cells. The production of molded arrays was fast and cost effective with a reasonable light output and uniformity. The light output and uniformity of the array were measured using a Hamamatsu MPPC in current mode and a collimated radioactive Sr-90 source mounted on a precision two dimensional scanning table [3]. The molded cell had a steep response at the edges and a smooth response through the central region (Fig. 1).The molded parts were less transparent and had residual stress, which may have contributed to their non-uniform response. Results indicated about 5\% optical crosstalk.

\section{Tests of Molded Cell Production}

Most of our molded cells were produced using a large capacity press. Because the volume of the cell is small, the large volume of raw material (polystyrene pellets and fluors) fed 
into the press was kept at high temperature for about thirty minutes or more. The longer the material is kept at high temperature, the more the transmittance of the material degenerates. A smaller

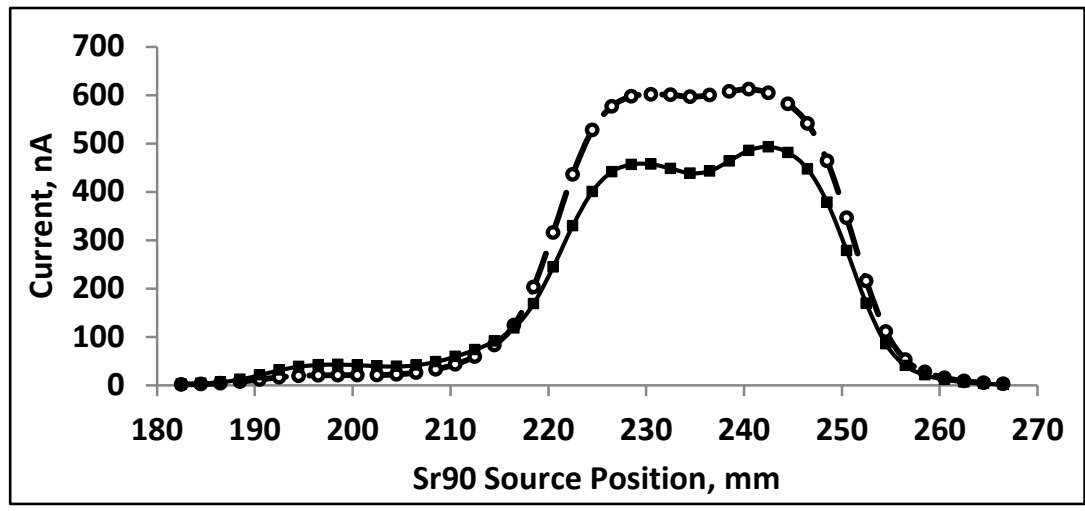

Fig. 1. Current response of $30 \times 30 \mathrm{~mm}^{2}$ square cells with $3 \mathrm{~mm}$ thickness scanned across the center line of a spherically dimpled cell. The molded polystyrene cell (closed squares) is in $2 \mathrm{x}$ 2 aray. The cast polyvinyltolulene single cell (open circles) has a milled dimple.

capacity press was subsequently used, with the time from injecting polystyrene pellets with fluors into the feeder to the ejection of a finished cell about five minutes. In addition, the low capacity press has better control of production parameters such as temperature and pressure. As a result, the transmittance of the molded cells made with this press was substantially improved. With the higher transmittance, the uniformity of cells with a $90 \%$ dimple depth showed visible overcompensation. Further optimized dimple depth will improve the uniformity of light response across the full area of the molded cell.

\section{New Diamond Cutter for a Spherical Dimple}

The quality of the dimple surface is a crucial factor for uniform response of a milled cell [3]. For producing cell arrays we have used a ball shaped bit installed at about $45^{\circ}$ to the cutting surface, such that the tip of the bit was out of the dimple [3]. A diamond cutter can be used to reduce or avoid melting of the dimple surface during milling and to obtain a finished surface. The spherical dimple surface can be produced by simultaneously rotating the diamond cutter in both vertical and horizontal planes and linearly moving the bit in space with deeper dimples using lower cutter vertical speed. This diamond cutter assembly was used for production of $9 \mathrm{x}$ 11 square cell array. Currently, the cutter assembly is instrumented with one diamond.

\section{IRL Assembly}

We observed a flat or uniform response across the cell area when the photo-detector was flush with the flat area on the dimpled side of the cell. That is, the sensor was adjacent to but not inside the dimple. Such a position requires a spacer between the existing electronics board and the photo sensor or the electronics board must be modified with an opening or additional mounting board to accommodate the photo detector. Because a spacer increases the thickness of the IRL assembly by $1 \mathrm{~mm}$, we started with a modification of the existing HBU2 electronics board $[1,2]$ by introducing the opening for the photo-detector based on an additional small 0.15 $\mathrm{mm}$ thick electronics board. Those small boards were mounted on the electronics board and then the IRL could be assembled and tested (Fig. 2). Connecting an array and an electronics board requires alignment holes in both. Because the electronics board is about $0.775 \mathrm{~mm}$ thick, the alignment pins were installed in the array and then connected to the board. We used two pins per array of 6 x 6 cells. For the each electronics boards we need four such arrays. When four arrays were connected to the board, the IRL assembly was structurally weak. We are investigating the structural stability of larger arrays ( $9 \times 11$ and $12 \times 12)$. 


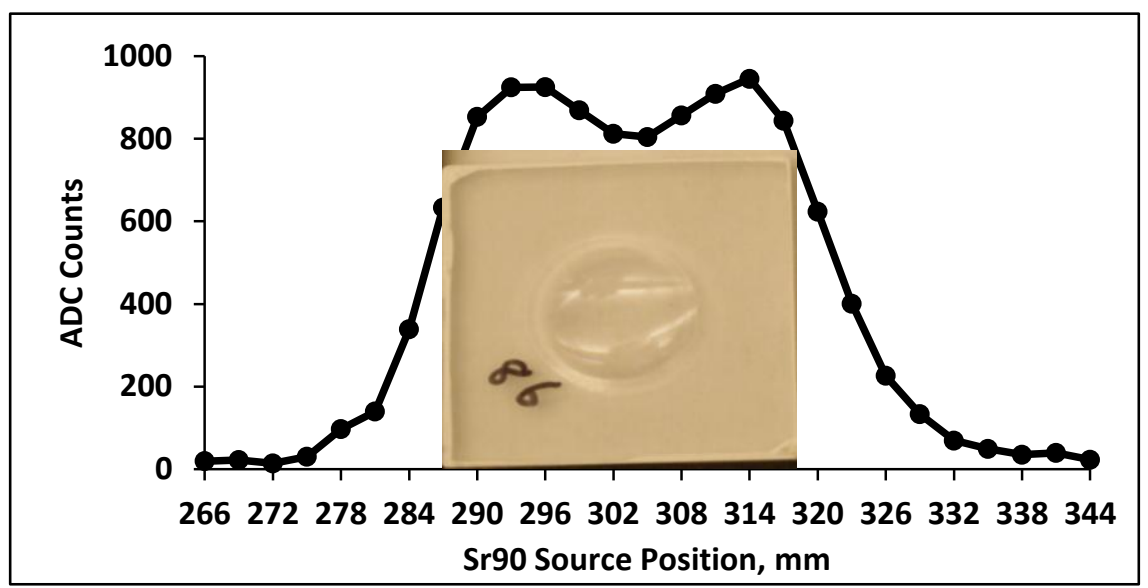

Fig. 2. Response of a single $30 \times 30 \mathrm{~mm}^{2}$ square cell of $3 \mathrm{~mm}$ thickness from an array of $6 \times 6$ cells in the IRL.

Performed with a radioactive source scanned across the center of the dimpled cell. The photograph illustrates the position of the response features.

\section{Summary}

The production by injection molding or machining of various dimpled cells and dimpled cell arrays all of $3 \mathrm{~mm}$ thickness was investigated.

The transmittance properties and production efficiency of molded cell have been improved and are now comparable with that of cast, machined cells.

The existing CALICE HBU2 electronics board was modified to accommodate SM MPPCs and arrays of dimpled cells. The composite IRL was tested using the SM LEDs in situ and a Sr90 source scan. Beam tests are in a planning stage.

\section{Acknowledgements}

The authors would like to thank the technicians and the engineers who contributed to the design and construction of the prototypes, Mark Niggemann (Dynasty Mold Builders) and Thomas J. Fitzpatrick (FNAL) for preparation of the apparatus. This work was supported in part by the US Department of Energy, the US National Science Foundation, and operated in part by Fermi Research Alliance, LLC under Contract No. De-AC02-07CH11359 with the United States Department of Energy.

\section{References}

[1] M. Reinecke, Performance of the Large Scale Prototypes of the CALICE Tile Hadron Calorimeter, IEEE Nucl. Science Symp. (NSS), N35-3, Seoul, Oct. 27 - Nov. 2, 2013

[2] O. Hartbrich and M. Terwort, Recent Advances of the Engineering Prototype of the CALICE Analog Hadron Calorimeter (2012) http://arxiv.org/pdf/1201.5264.pdf

[3] G. Blazey, et al., Nucl. Instr. and Meth. A605 (2009) 277.

[4] F. Abu-Ajamieh, et al., Nucl. Instr. and Meth. A659 (2011) 348.

[5] F. Abu-Ajamieh, et al., Physics Procedia 37 (2012) 789 